\title{
Multiscaling in the randomly forced and conventional Navier-Stokes equations
}

\author{
Anirban Sain ${ }^{\mathrm{a}}$, Rahul Pandit ${ }^{\mathrm{b}, *, 1}$ \\ ${ }^{a}$ Department of Physics, Simon Fraser University, Burnaby, BC, Canada V5A 1 S6 \\ ${ }^{\mathrm{b}}$ Department of Physics, Indian Institute of Science, Bangalore 560 012, India
}

\begin{abstract}
We present an overview of some results we have obtained recently (A. Sain, Manu and R. Pandit, Phys. Rev. Lett. 81 (1998) 4377) from a pseudospectral study of the randomly forced Navier-Stokes equation (RFNSE) stirred by a stochastic force with zero mean and a variance $\sim k^{4-d-y}$, with $k$ the wavevector and the dimension $d=3$. These include the multiscaling of velocity structure functions for $y \geqslant 4$ and a demonstration that the multiscaling exponent ratios $\zeta_{p} / \zeta_{2}$ for $y=4$ are in agreement with those obtained for the Navier-Stokes equation forced at large spatial scales ( $3 d \mathrm{NSE}$ ). We also study a coarse-graining procedure for the $3 d \mathrm{NSE}$ and examine why it does not lead to the RFNSE.
\end{abstract}

Keywords: Fluid turbulence; Navier-Stokes equation; Randomly forced Navier-Stokes equation

\section{Introduction}

The development of an understanding of the scaling behaviour of the velocity $\mathbf{v}$ structure functions $\mathscr{S}_{p}(r) \equiv\left\langle\left|\mathbf{v}_{i}(\mathbf{x}+\mathbf{r})-\mathbf{v}_{i}(\mathbf{x})\right|^{p}\right\rangle$, where the angular brackets denote an average over the statistical steady state, has been a central problem in homogeneous, isotropic fluid turbulence ever since Kolmogorov's classic study (K41) [2,3]. At large Reynolds numbers $R e$, his elegant dimensional considerations suggested that, for $r \equiv|\mathbf{r}|$ in the inertial range, which lies between the forcing scale $L$ and the dissipation scale

\footnotetext{
in It is a great pleasure for us to contribute this paper to these Proceedings in honour of Professor C.K. Majumdar. He has always been a source of great inspiration for all of us. We wish him many years of active involvement in research and teaching.

* Corresponding author.

E-mail address: rahul@physics.iisc.ernet.in (R. Pandit)

${ }^{1}$ Also at Jawaharlal Nehru Centre for Advanced Scientific Research, Bangalore, India.
} 
$\eta_{d}$, these structure functions scale as $\mathscr{S}_{p} \sim r^{\zeta_{p}}$, with $\zeta_{p}=p / 3$. Experiments [4,5] find instead that multiscaling obtains, namely, $\zeta_{p}$ is a nonlinear, monotonically increasing function of $p$ that lies significantly below the K41 value $\zeta_{p}^{K 41}=p / 3$ for $p>3$; this has also been borne out by numerical studies of the three-dimensional NavierStokes equation for an incompressible fluid forced at large spatial scales ( $3 d \mathrm{NSE}$ ) [4-6]. Experimental and numerical data for these exponents are well parametrised by the She-Leveque [7] (SL) formula $\zeta_{p}^{S L}=(p / 9)+2\left[1-(2 / 3)^{p / 3}\right]$, which we use below.

The calculation of the multiscaling exponents $\zeta_{p}$ for the $3 d \mathrm{NSE}$ has, so far, proved to be analytically intractable with realistic external forcing at large spatial scales. However, some analytical studies, which use techniques of statistical field theory, have been possible for a variant of this equation in which a Gaussian random force acts on all length scales. We refer to this as the randomly forced Navier-Stokes equation (RFNSE). In wavevector or Fourier space (henceforth $k$ space) the RFNSE is

$$
\partial_{t} \mathbf{v}_{i}(\mathbf{k})+i M_{i j k}(\mathbf{k}) \sum_{\mathbf{q}} \mathbf{v}_{j}(\mathbf{q}) \mathbf{v}_{k}(\mathbf{k}-\mathbf{q})=-v k^{2} \mathbf{v}_{i}(\mathbf{k})+\mathbf{f}_{i}(\mathbf{k}),
$$

where time $t$ arguments of $\mathbf{v}$ and $\mathbf{f}$ have been suppressed for notational convenience, $\mathbf{k}$ and $\mathbf{q}$ denote wave vectors, $i, j, l$ Cartesian components, $M_{i j l}(\mathbf{k}) \equiv\left[k_{j} P_{i l}(\mathbf{k})+\right.$ $\left.k_{l} P_{i j}(\mathbf{k})\right] / 2$, and $P_{i j}(\mathbf{k}) \equiv\left[\delta_{i j}-k_{i} k_{j} / k^{2}\right]$ the transverse projector, which enforces the incompressibility condition. Fourier transforms are implied by wavevector arguments and the statistics of the random force $\mathbf{f}(\mathbf{k}, t)$ is $\langle\mathbf{f}(\mathbf{k}, t)\rangle=0$ and $\left\langle\mathbf{f}_{i}(\mathbf{k}, t) \mathbf{f}_{j}\left(\mathbf{k}^{\prime}, t^{\prime}\right)\right\rangle=$ $A k^{4-d-y} P_{i j}(\mathbf{k}) \delta\left(\mathbf{k}+\mathbf{k}^{\prime}\right) \delta\left(t-t^{\prime}\right)$, where $t$ and $t^{\prime}$ are times and $d$ the dimensions $(d=3$ henceforth unless specified otherwise); this is consistent with the homogeneity, isotropy, and incompressibility conditions.

There have been some approximate analytical studies of this RFNSE. Those that use the dynamical renormalization-group (DRG) technique at the one-loop level [8,9,24,25] obtain recursion relations to $\mathcal{O}(y)$ in a small- $y$ expansion. These yield an infrared-stable fixed point for $y>0$ and the K41 scaling form for the energy spectrum, namely, $E(k) \sim k^{2}|\mathbf{v}(\mathbf{k})|^{2} \sim k^{-5 / 3}$ if $y=4$. The one-loop predictions for some universal amplitudes and amplitude ratios like the Kolmogorov constant, the skewness factor $\left(-\left\langle\left(\partial_{x} v_{x}\right)^{3}\right\rangle /\left\langle\left(\partial_{x} v_{x}\right)^{2}\right\rangle^{3 / 2}\right)$, etc., are also close to experimental results. This is surprising because the approximations used in this DRG have been criticised [10,11,26] since they set $y=4$ in a small- $y$ expansion and neglect an infinite number of marginal operators (at $y=y_{c}=4$ ). An $N$-component generalisation of the RFNSE has also been explored and, in the limit $N \rightarrow \infty[10,26]$, it has been argued that $E(k) \sim k^{-3 / 2}$ for $y \geqslant y_{c}=4$. Thus, for $N \rightarrow \infty$ and at the level of $E(k), y_{c}=4$ is the analogue of the upper critical dimension in equlibrium critical phenomena. An early numerical study [12] of the RFNSE had obtained $E(k) \sim k^{-5 / 3}$, but, until our recent work [1], there had been no studies of the multiscaling exponents $\zeta_{p}$ for the RFNSE with $p>2$. Nor had there been attempts to see how high-intensity vortical structures, which form filaments in the $3 d \mathrm{NSE}[4,5,13,14]$, organise themselves in the RFNSE; such filaments are also seen in experiments [15,27]. 
It was against this background that we carried out a direct numerical study (DNS) of the RFNSE. We present a summary of the results of this study in the next section. One of our main results is that, at the level of extended self similarity (ESS) [16] in which the exponent ratios $\zeta_{p} / \zeta_{q}$ are determined from $\log$-log plots of $\mathscr{S}_{p}$ versus $\mathscr{S}_{q}$, the RFNSE with $y=4$ yields $3 d$ NSE-type multiscaling. (This procedure is called extended self similarity since it extends the apparent inertial range over which inertial-range multiscaling exponents can be fit $[16,17]$.) Given this, it is natural to ask whether a coarse-graining procedure can be used to map the $3 d$ NSE onto the RFNSE. Such a coarse-graining programme has been used to map the deterministic, but spatiotemporally chaotic, Kuramoto-Sivashinsky (KS) equation onto the stochastic Kardar-Parisi-Zhang (KPZ) equation in both one [18,19] and two [20] dimensions; thus we expect the long-distance and large-time behaviours of their correlation functions to be the same. In Section III we try to carry out a similar coarse graining of the $3 d \mathrm{NSE}$ and show that it does not yield the RFNSE. The reasons for this are instructive and they are examined in some detail as is the relation of our coarse-grained equation to another effective equation [21] for fluid turbulence.

\section{RFNSE Results}

We have studied the RFNSE by a numerical pseudospectral method whose details are described in Refs. [1,22]. This uses $64^{3}$ or, in some cases, $80^{3}$ grids in a cubic box of linear size $L=2 \pi$ with periodic boundary conditions. We include both a viscosity $v$ and a hyperviscosity $v_{H}$, i.e., our dissipation term is $\left(v+v_{H} k^{2}\right) k^{2} \mathbf{v}(\mathbf{k})$. The exponents $\zeta_{p}$ are unaffected by $v_{H}$ especilly if $v>0$ [17,23]. For a fixed grid, the Taylor-microscale Reynolds numbers $R e_{\lambda}$ that obtains in a DNS of the RFNSE is larger than in the $3 d$ NSE ( $R e_{\lambda} \simeq 120$ compared to $R e_{\lambda} \simeq 22$ in our study). [1]; thus the inertial range is larger too. However, $R e_{\lambda}$ fluctuates more in the RFNSE (inset of left panel of Fig. 1), so longer runs are required for reliable averages. Details of our averaging times, etc., are given in Refs. [1,22]. We also emphasize that, in spite of the delta-correlated stochastic force in the RFNSE, the temporal variation of $\mathbf{v}(\mathbf{k})$ is similar to that in the $3 d$ NSE. The modes $\mathbf{v}(\mathbf{k})$ fluctuate rapidly as $k$ increases. The time scale of their fluctuation is the scale-dependent eddy-turn-over time $\tau_{k} \sim(\bar{\varepsilon})^{-1 / 3} k^{-2 / 3}$. We have checked the existence of this hierarchy of times in both the $3 d$ NSE and the RFNSE [22]. In the latter, the stochastic force puts a high-frequency ripple on $\mathbf{v}(\mathbf{k})$ even for small $k$, but this does not affect its overall variation significantly nor the exponent ratios if $y=4$.

We are now in a position to summarise our principal results for the RFNSE: We find that the velocity structure functions $\mathscr{S}_{p}(r)$ and their $k$-space analogues $S_{p}(k) \equiv$ $\left\langle|\mathbf{v}(\mathbf{k})|^{p}\right\rangle$ display multiscaling for $y \geqslant 4$. Log-log plots of $S_{2}(k)$ versus $k$ (Fig. 1) yield $\zeta_{2}^{\prime}$ since $S_{2}(k) \sim k^{-\zeta_{2}^{\prime}}$ in the inertial range; the $r$-space exponent $\zeta_{2}$ follows [1] from $\zeta_{2}=\zeta_{2}^{\prime}-3$. (We use the convention that exponents of $k$-space structure functions have a prime; real-space ones do not.) Our data with a $64^{3}$ grid for $y \geqslant 4$ are consistent with $\zeta_{2}^{\prime}=11 / 3$, the K41 value, since $E(k) \sim k^{2} S_{2}(k) \sim k^{-5 / 3}$ (for $y=4$ see also 

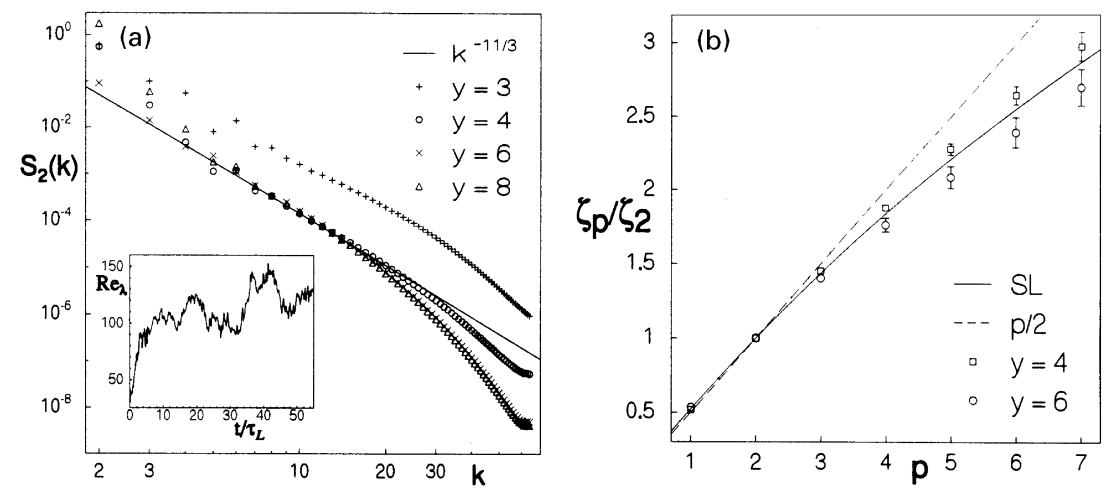

Fig. 1. Left panel: Log-log plots (base 10) of $S_{2}(k)$ versus $k$ for different values of $y$. The line indicates the K41 result $S_{2}(k) \sim k^{-11 / 3}$. $\mathbf{k}$ indicates the shell number, which is twice the wave number $\left(=\frac{2 \pi}{L} n\right)$. Inset shows a repesentative plot of $\operatorname{Re}_{\lambda}$ versus time $(t)$ for $y=4$. Right panel: Inertial-range exponent ratios $\zeta_{p} / \zeta_{2}$ versus $p$ for the RFNSE with $y=4$ and 6 ; the line indicates the SL formula.

Ref. [12]); the top panel of Fig. 2 shows, via a $\log -\log$ plot of $E(k)$ versus $k$ for an $80^{3}$ grid and $y=4$, that our result for $\zeta_{2}^{\prime}$ is not affected by using a finer grid. We determine the ratios $\zeta_{p} / \zeta_{2}$ by using the ESS procedure [16,17] mentioned above; a representative $\log$-log ESS plot of $\mathscr{S}_{4}$ versus $\mathscr{S}_{2}$ is shown in the middle panel of Fig. 2 for a grid with $80^{3}$ points. The exponent ratios $\zeta_{p} / \zeta_{2}$, which follow from the inertial-range slopes of such plots, are shown as functions of $p$ in the bottom panel of Fig. 2 (for an $80^{3}$ grid, $y=4$, and $p \leqslant 4$ ) and in the right panel of Fig. 1 (for a $64^{3}$ grid, $y=4$ and $y=6$, and $\left.p \leqslant 7\right)$. We see then that, for $y=4$, the exponent ratios are close to the $3 d \mathrm{NSE}$ result (parametrised here by the She-Leveque (SL) result shown by the full line) at least for $p \leqslant 7$. The qualitative behaviours of the probability distributions $P\left(\delta v_{\alpha}(r)\right)$, where $\delta v_{\alpha}(r) \equiv v_{\alpha}(\mathbf{x})-v_{\alpha}(\mathbf{x}+\mathbf{r})$, are also similar in the two models; a representative plot is shown in Fig. 3. These probabilty distributions have non-Gaussian tails, for $r$ in the dissipation ranger; and the deviations from a Gaussian form for $y=4$ and $y=6$ are roughly the same if comparable values of $r / \eta_{d}$ are used ( $\eta_{d}$ is the Kolmogorov dissipation scale). As we have noted in Ref. [1], longer runs with finer grids are required to settle conclusively whether the ratios $\zeta_{p} / \zeta_{2}$ are actually different for $y=4$ and $y=6$ (Fig. 1).

Constant- $|\omega|$ surfaces, where $\omega$ is the vorticity, are markedly different as we have discussed elsewhere $[1,22]$; the stochastic force destroys the filamentary structures that obtain in $3 d$ NSE studies $[5,13,14,22]$. The smaller $y$ is the more efficient this destruction: for $y=4$ one can hardly make out filaments but they start reappearing [22] faintly around $y=6$. This has implications for the She-Leveque (SL) [7] formula for $\zeta_{p}$, which uses the codimension of such structures as an important input.

Thus the RFNSE with $y=4$ seems to be in the same universality class as the $3 d$ NSE at least in so far as our RFNSE exponent ratios are within error bars of those for the $3 d$ NSE (Fig. 1). Note though that the RFNSE with $y=4$ falls in the same universality class as the $3 d$ NSE only at the level of ESS. For $y=4$ the energy flux through the $k^{\text {th }}$ 

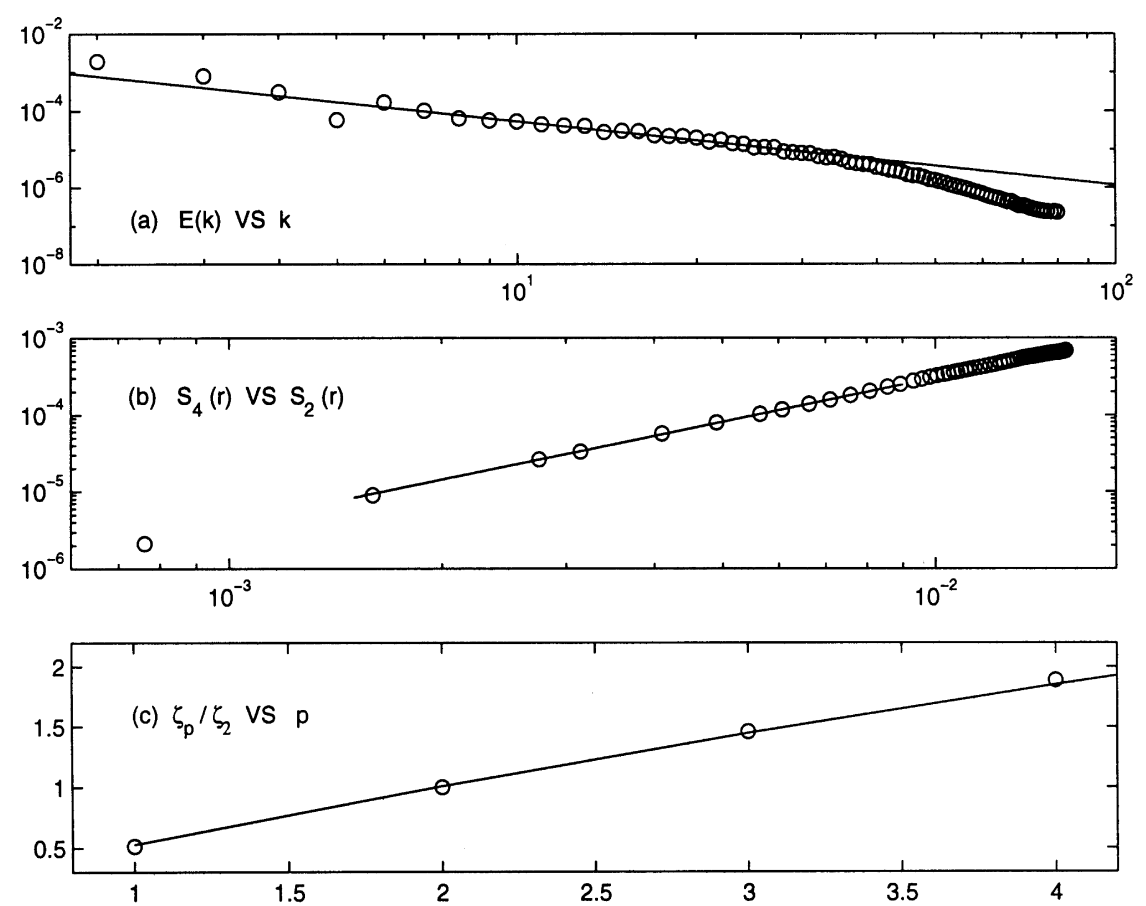

Fig. 2. Representative plots obtained from our RFNSE run, carried out on an $80^{3}$ grid, with $y=4$. Data have been averaged over $\sim 2.5 \tau_{L}$, the box-size eddy turnover time. (a) $\log -\log$ plot of $E(k)$ versus $k$; the solid line represents $k^{-5 / 3}$ scaling. (b)An ESS $\log$ - $\log$ plot of $\mathscr{S}_{4}(r)$ versus $\mathscr{S}_{2}(r)$; the solid line represents a regression fit. (c) Multiscaling exponent ratios $\zeta_{p} / \zeta_{2}$ versus $p$; the solid line represents the SL formula (see text).

shell $\Pi_{k} \sim \log (k L)$ in the RFNSE [1,3], whereas, in the $3 d \mathrm{NSE}, \Pi_{k}=$ constant. Thus all correlation functions in these two models do not have the same behaviours in the inertial range. These weak deviations must cancel in the ratios of structure functions since our ESS procedure works and yields, for $y=4$, ratios $\zeta_{p} / \zeta_{2}$ that are within error bars of the SL result for the $3 d$ NSE.

\section{Numerical coarse graining of the Navier-Stokes equation}

We have shown above that the RFNSE yields the same multiscaling exponent ratios as the $3 d \mathrm{NSE}$ if $y=4$. In view of this it is natural to ask whether we can obtain the RFNSE from the $3 d$ NSE by a coarse-graining procedure that retains inertial-range scales. The general issue of interest here is the following: The $3 d$ NSE is a deterministic partial differential equation (PDE) which shows chaotic behaviour. Can we find a stochastic PDE in which the external noise mimics the deterministically generated chaos to the extent that correlation functions show the same long-distance and long-time behaviours? The RFNSE is a likely candidate for such a stochastic PDE. 


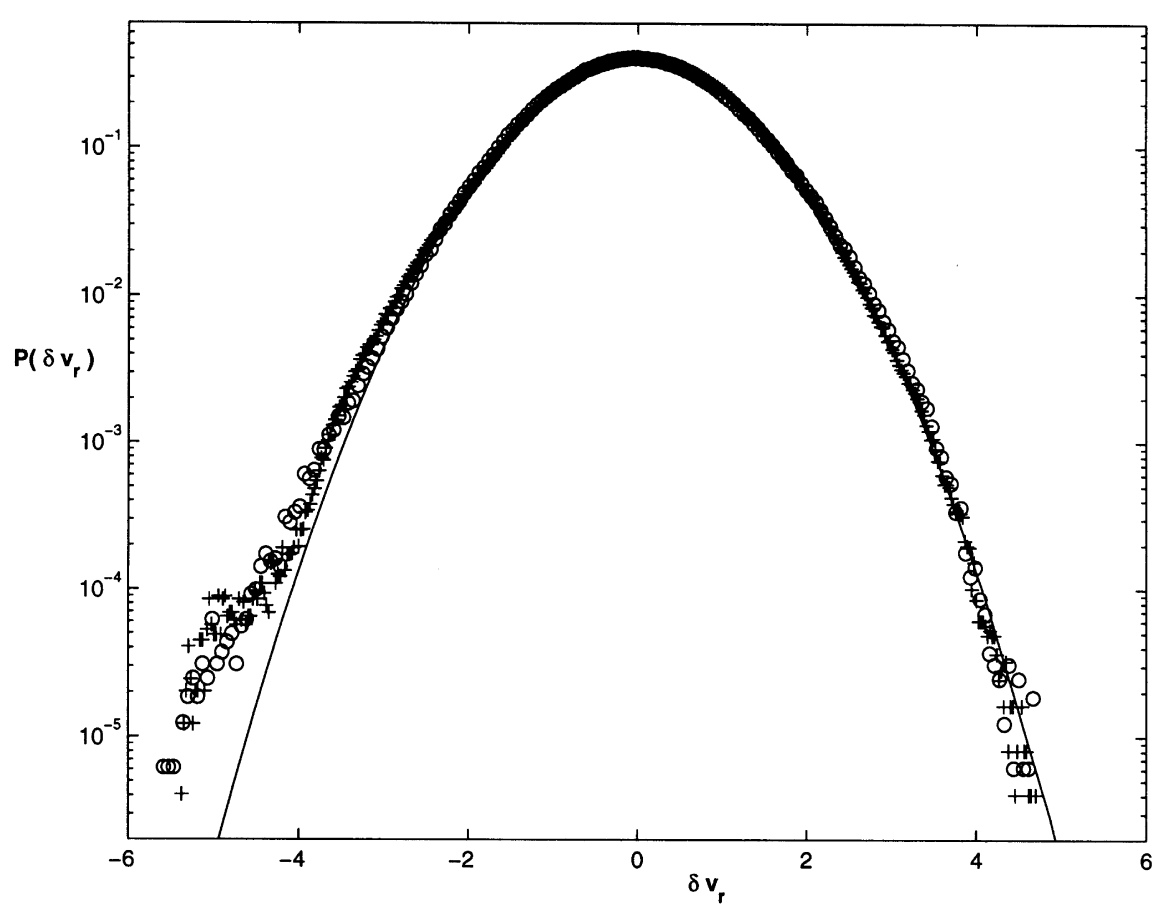

Fig. 3. Semilog plots of the distribution function $P\left(\delta v_{r}\right)$ (i.e., $P\left(\delta v_{\alpha}(r)\right)$ averaged over $\left.\alpha\right)$ versus $\delta v_{r}$, for $r$ close to the dissipation range and for $y=4(\mathrm{o})$ and $6(+)$. A Gaussian distribution (solid line) is shown for comparison. We choose $r=5$ for $y=4$, and $r=10$ for $y=6$, which correspond to $r / \eta_{d}=7.65$ for $y=4$ and $r / \eta_{d}=6.25$ for $y=6$, where $\eta_{d}$ is the Kolmogorov dissipation scale. We picked the values of $r / \eta_{d}$ for $y=4$ and $y=6$ to be as close as possible in our runs in order to get as meaningful a comparison of the distributions as possible.

Such a programme has been carried out for the Kuramoto-Sivashinsky (KS) equation [18-20] where an explicit, numerical coarse-graining procedure has shown that this equation is in the universality class of the Kardar-Parisi-Zhang (KPZ) equation in spatial dimensions $d=1$ and 2 .

Our coarse-graining scheme for the $3 d \mathrm{NSE}$ employs the following strategy: Since we want to investigate the universal multiscaling of velocity structure functions, we divide the velocity modes into those in the inertial range and those outside it. We then integrate out the latter by summing over the modes which are externally driven and the ones which fall in the dissipation range; the reduced system that we are left with (see below) consists of only the inertial-range modes. Our aim is to obtain an effective stochastic equation which will correctly describe the multiscaling of structure functions in the inertial range $\mathscr{I}$ and over the associated time scales (see below). Our starting point is the $k$-space $3 d \mathrm{NSE}$

$$
\partial v_{i}(\mathbf{k}) / \partial t=-v k^{2} v_{i}(\mathbf{k})-i M_{i m n}(\mathbf{k}) \sum_{\mathbf{q}} v_{m}(\mathbf{q}) v_{n}(\mathbf{k}-\mathbf{q})+f_{i}(\mathbf{k}) \theta\left(k_{0}-k\right),
$$




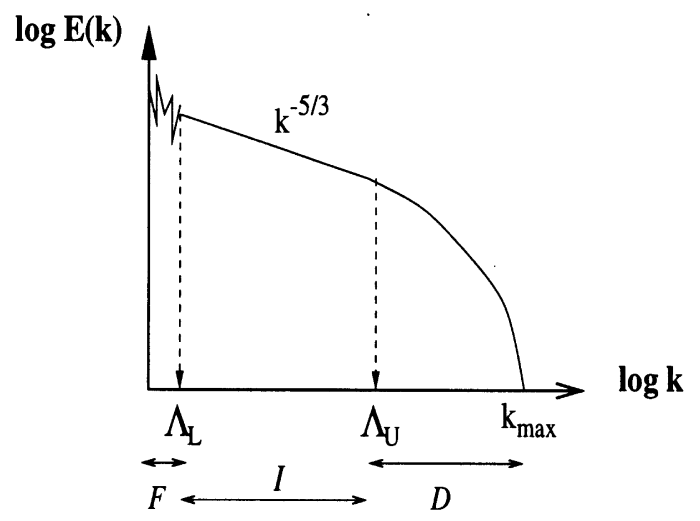

Fig. 4. The various ranges of scales $\mathscr{F}$ (forcing), $\mathscr{I}$ (inertial), and $\mathscr{D}$ (dissipation) involved in our coarse-graining procedure are indicated in this schematic plot of the energy spectrum.

where the step function $\theta$ ensures that only modes with wavevector magnitudes upto $k_{0}$ are forced externally. In terms of the discretised $k$-space used in our DNS, only the first two $k$ shells with $k<1.5$ are forced. We work with $\mathbf{v}(\mathbf{k}=0)=0$ without any loss of generality. The inertial range $\mathscr{I}$ is $2 \pi / L \ll k \ll k_{d}$, where $k_{d}$ is the inverse of the Kolmogorov dissipation scale $\left(k_{d} \sim\left(\bar{\varepsilon} / v^{3}\right)^{1 / 4}\right)$.

Since our coarse-graining procedure integrates out the modes $\mathbf{v}(\mathbf{k})$ with $k<\Lambda_{L}$ and $k>\Lambda_{U}$, the effective equation we obtain below is valid only for time scales $\tau \in$ $\left[\tau_{U}, \tau_{L}\right]$, where $\tau_{L}$ and $\tau_{U}$ are the eddy-turn-over times associated, respectively, with the wavevector scales $\Lambda_{L}$ and $\Lambda_{U}$ shown schematically in Fig. 4. We define three distinct wave-number ranges: $\mathscr{F} \equiv\left[0, \Lambda_{L}\right), \mathscr{I} \equiv\left[\Lambda_{L}, \Lambda_{U}\right]$, and $\mathscr{D} \equiv\left(\Lambda_{L}, k_{\max }\right]$; to make these definitions consistent with the conventional definition of the inertial range we must choose $\Lambda_{L} \sim 2 \pi / L$ and $\Lambda_{U} \sim k_{d}$.

It is useful to write the nonlinear term in Eq. (2) as a sum of five terms as shown below:

$$
\begin{aligned}
-i M_{i m n}(\mathbf{k}) \sum_{\mathbf{q}} v_{m}(\mathbf{q}) v_{n}(\mathbf{k}-\mathbf{q})= & T_{1}+T_{2}+T_{3}+T_{4}-i M_{i m n}(\mathbf{k}) \\
& \times \sum_{\mathbf{q}, \mathbf{k}-\mathbf{q} \in \mathscr{I}} v_{m}(\mathbf{q}) v_{n}(\mathbf{k}-\mathbf{q}),
\end{aligned}
$$

with

$$
\begin{aligned}
& T_{1}=-i M_{i m n}(\mathbf{k}) \sum_{\mathbf{q}, \mathbf{k}-\mathbf{q} \in \mathscr{F}} v_{m}(\mathbf{q}) v_{n}(\mathbf{k}-\mathbf{q}) ; \\
& T_{2}=-i M_{i m n}(\mathbf{k}) \sum_{\mathbf{q}, \mathbf{k}-\mathbf{q} \in \mathscr{D}} v_{m}(\mathbf{q}) v_{n}(\mathbf{k}-\mathbf{q}) ; \\
& T_{3}=-i M_{i m n}(\mathbf{k}) \sum_{\mathbf{q} \in(\mathscr{F} \text { or } \mathscr{D}) ; \mathbf{k}-\mathbf{q} \in \mathscr{I}} v_{m}(\mathbf{q}) v_{n}(\mathbf{k}-\mathbf{q}) ; \\
& T_{4}=-i M_{i m n}(\mathbf{k}) \sum_{\mathbf{q} \in \mathscr{F}, \mathbf{k}-\mathbf{q} \in \mathscr{D}} v_{m}(\mathbf{q}) v_{n}(\mathbf{k}-\mathbf{q}) .
\end{aligned}
$$


The fluctuation time scales of each one of the terms in the sums in $T_{1}-T_{4}$ are controlled by the faster one of the modes $v_{m}(\mathbf{q})$ and $v_{n}(\mathbf{k}-\mathbf{q})$. Thus $T_{1}$ is essentially constant since both $\mathbf{q}$ and $\mathbf{k}-\mathbf{q} \in \mathscr{F}$ and the associated modes vary very slowly for the times $\tau_{U}<\tau<\tau_{L}$ of interest for our effective equation. By contrast, $T_{2}$ fluctuates rapidly since both $\mathbf{q}$ and $\mathbf{k}-\mathbf{q} \in \mathscr{D}$ here, so, as we elaborate below, it can be thought of as an additive noise. The fluctuation time scales of the terms $T_{3}$ and $T_{4}$ lie in between those of $T_{1}$ and $T_{2}$. Our coarse-graining procedure leads to the following effective equation for the modes $\mathbf{v}(\mathbf{k})$ with $\mathbf{k} \in \mathscr{I}$ and $\tau \in\left[\tau_{U}, \tau_{L}\right]$ :

$$
\partial v_{i}(\mathbf{k}) / \partial t=-v_{i j l m} k_{j} k_{m} v_{l}(\mathbf{k})-i M_{i m n}(\mathbf{k}) \sum_{\mathbf{q}, \mathbf{k}=\mathbf{q} \in \mathscr{I}} v_{m}(\mathbf{q}) v_{n}(\mathbf{k}-\mathbf{q})+F_{i}^{A}(\mathbf{k})+T_{3} .
$$

Here $v_{i j l m}(k)$ is the effective viscosity tensor that is generated; we give an expression for it below. The term $F_{i}^{A}(\mathbf{k})$ is an effective additive forcing parts of which fluctuate rapidly; these parts can, therefore, be interpreted as an additive noise. Specifically

$$
F_{i}^{A}(\mathbf{k})=T_{1}+T_{2}+T_{4}-v k^{2} v_{i}(\mathbf{k})+v_{i j l m} k_{j} k_{m} v_{l}(\mathbf{k}) .
$$

The term $T_{3}$ can be interpreted as an effective multiplicative forcing part of which fluctuates rapidly; this part can, therefore, be interpreted as a multiplicative noise. If we write

$$
T_{3}=-i M_{i m n}(\mathbf{k}) \sum_{\mathbf{q} \in(\mathscr{F} \text { or } \mathscr{D}), \mathbf{k}-\mathbf{q} \in \mathscr{I}} F_{m}^{M}(\mathbf{q}) v_{n}(\mathbf{k}-\mathbf{q}),
$$

then

$$
F_{i}^{M}(\mathbf{q})=v_{i}(\mathbf{q}), \quad \mathbf{q} \in(\mathscr{F} \text { or } \mathscr{D}) .
$$

We now separate the slow and rapidly fluctuating parts of $F_{i}^{A}$ and $F_{i}^{M}$ as follows. We define $F_{i}^{A}=\left\langle F_{i}^{A}\right\rangle+\delta F_{i}^{A}$ and $F_{i}^{M}=\left\langle F_{i}^{M}\right\rangle+\delta F_{i}^{M}$, with

$$
\begin{aligned}
& \left\langle F_{i}^{A}(\mathbf{k})\right\rangle=T_{1}, \\
& \delta F_{i}^{A}(\mathbf{k})=-v k^{2} v_{i}(\mathbf{k})+v_{i j l m} k_{j} k_{m} v_{l}(\mathbf{k})+T_{2}+T_{4}, \\
& \left\langle F_{i}^{M}(\mathbf{q})\right\rangle=v_{i}(\mathbf{q}) \theta\left(\Lambda_{L}-q\right), \\
& \delta F_{i}^{M}(\mathbf{q})=v_{i}(\mathbf{q}) \theta\left(q-\Lambda_{U}\right) .
\end{aligned}
$$

The angular brackets in Eqs. (9)-(12) denote averages over times $\sim \tau_{U}$ over which $v_{i}(\mathbf{q})$, for which $\mathbf{q} \in \mathscr{F}$, is essentially constant. This decomposition assumes that the fluctuating parts of $F_{i}^{A}$ and $F_{i}^{M}$ have zero mean. For $\delta F_{i}^{M}$ (from Eq. (12)) this requires $\left\langle v_{i}(\mathbf{q})\right\rangle=0$, for $q>\Lambda_{U}$, when averaged over a time $\sim \tau_{U}$. This is satisfied since it follows from homogeneity that $\langle\mathbf{v}(\mathbf{k})\rangle=0$ for all $\mathbf{k}$, if it is averaged over the associated eddy-turn-over-time $\tau_{k}$. For $\delta F_{i}^{A}$ (Eq. (10)), the first two terms which are proportional to $v_{i}(\mathbf{k})$ give zero when averaged over times $\sim \tau_{k}$. We also require $\left\langle T_{2}+T_{4}\right\rangle=0$. We assume separately that $\left\langle T_{2}\right\rangle=0$ and $\left\langle T_{4}\right\rangle=0$. This is plausible as far as $T_{2}$ is 
concerned since it contains only modes from $\mathscr{D}$ that are faster than modes in $\mathscr{I}$ (Eq. (12)). $\left\langle T_{4}\right\rangle$ is identically zero for $k \ll \Lambda_{U}$. This is because, only for $k \lesssim \Lambda_{U}$, can we construct a few terms that contribute to $T_{4}$ such that $q \leqslant \Lambda_{L}$ and $|\mathbf{k}-\mathbf{q}| \geqslant \Lambda_{U}$ (since $\left.\Lambda_{U} / \Lambda_{L} \gg 1\right)$. For $k \ll \Lambda_{U}$, the region in which we are interested, there are no $T_{4}$-type terms.

If $\delta F_{i}^{A}(\mathbf{k})$ and $\delta F_{i}^{M}(\mathbf{k})$ are to mimic external noises in Eq. (5), for the inertial-range modes, they must (a) fluctuate more rapidly than the $\mathscr{I}$ modes (i.e, faster than $\tau_{U}^{-1}$ ) and (b) obey suitable causality conditions. Condition (a) is basically satisfied since $T_{2}, T_{4}$ (Eq. (10)), and $\delta F_{i}^{M}$ (Eq. (12)) contain at least one velocity mode from $\mathscr{D}$, so they fluctuate faster than the nonlinear term in Eq. (5). We impose the causality conditions $^{2}$

$$
\begin{aligned}
& \left\langle\delta F_{i}^{A}(\mathbf{k}, t) v_{n}(-\mathbf{k}, t-s)\right\rangle=0, \quad \mathbf{k} \in \mathscr{I}, \\
& \left\langle\delta F_{i}^{M}\left(\mathbf{k}^{\prime}, t\right) v_{n}(\mathbf{k}, t-s)\right\rangle=0, \quad \mathbf{k}^{\prime} \in \mathscr{D}, \quad \mathbf{k} \in \mathscr{I},
\end{aligned}
$$

at least for sufficiently large $s$. Eq. (14) is satisfied because $\delta F_{i}^{M}\left(\mathbf{k}^{\prime}\right)=v_{i}\left(\mathbf{k}^{\prime}\right)$ is a faster mode than $v_{n}(\mathbf{k})$, so, when we average over the origin $t$ for a time $\sim \tau_{U}$, the product vanishes. If we substitute the expression for $\delta F_{i}^{A}$ (Eq. (10)) into Eq. (13) we get the following expression for $v_{i j l m}(\mathbf{k})$ :

$$
\begin{array}{r}
v k^{2}\left\langle v_{i}(\mathbf{k}, t) v_{n}(-\mathbf{k}, t-s)\right\rangle+v_{i j l m} k_{j} k_{m}\left\langle v_{l}(\mathbf{k}, t) v_{n}(-\mathbf{k}, t-s)\right\rangle \\
-i M_{i l m}(\mathbf{k}) \sum_{\mathbf{q}, \mathbf{k}-\mathbf{q} \in \mathscr{D}}\left\langle v_{l}(\mathbf{q}, t) v_{m}(\mathbf{k}-\mathbf{q}, t) v_{n}(-\mathbf{k}, t-s)\right\rangle=0 .
\end{array}
$$

Since our turbulent (statistical) steady state is isotropic and homogeneous we must have

$$
\left\langle v_{i}(\mathbf{k}, t) v_{n}(-\mathbf{k}, t-s)\right\rangle \equiv P_{i n}(\mathbf{k}) A(k, s),
$$

and

$$
\begin{gathered}
\sum_{\mathbf{q}, \mathbf{k}-\mathbf{q} \in \mathscr{D}}\left\langle v_{l}(\mathbf{q}, t) v_{m}(\mathbf{k}-\mathbf{q}, t) v_{n}(-\mathbf{k}, t-s)\right\rangle \equiv \\
C(k, s) \delta_{l m} k_{n}+D(k, s)\left(\delta_{l n} k_{m}+\delta_{m n} k_{l}\right) \\
+H(k, s) k_{l} k_{m} k_{n}
\end{gathered}
$$

which define the functions $A, C, D$, and $H$; note that these functions depend only on $k=|\mathbf{k}|$ and the time separation $s$. Furthermore, isotropy yields

$$
v_{i j l m}=c_{1} \delta_{i j} \delta_{l m}+c_{2} \delta_{i l} \delta_{j m}+c_{3} \delta_{i m} \delta_{l j},
$$

which gives, in conjunction with Eq. (15),

$$
c_{2} P_{\text {in }}(\mathbf{k}) k^{2} A(k, s)=v k^{2} P_{\text {in }}(\mathbf{k}) A(k, s)+\frac{i}{2} P_{\text {in }}(\mathbf{k}) k^{2} D(k, s),
$$

\footnotetext{
$\overline{{ }^{2} \text { One can think }}$ of more general causality conditions but these suffice for our purpose here and lead to an expression for the viscosity tensor.
} 
whence we get the effective shear viscosity $c_{2} \equiv v+\Delta v$ with $^{3}$

$$
\Delta v(k) \equiv \frac{i}{2} D(k, s) / A(k, s) .
$$

For our coarse-graining procedure to be valid, $\Delta v(k)$ should approach an $s$-independent value for $\tau_{U}<s<\tau_{L}$. However, this correction to the viscosity turns out to be very small here in contrast to what happens while coarse-graining the KS equation to obtain the KPZ equation [20]. The reason is as follows: $\Delta v(k)$ is proportional to $\frac{\langle v(\mathscr{D}) v(\mathscr{D}) v(\mathscr{T})\rangle}{\langle v(\mathscr{I}) v(\mathscr{I})\rangle}$, where $v(\mathscr{D})$ denotes a mode with $\mathbf{k} \in \mathscr{D}$, etc. Since the $v(\mathscr{D})$ 's are exponentially damped compared to the $v(\mathscr{I})$ 's the correction $\Delta v(k)$ is also small. In particular, our coarse-graining procedure also generates the terms $\left\langle F_{i}^{A}\right\rangle$ and $\left\langle F_{i}^{M}\right\rangle$, which turn out to be far larger than $\Delta v k^{2} v_{i}(\mathbf{k})$. This is again unlike what happens in the $\mathrm{KS} \rightarrow \mathrm{KPZ}$ mapping [20], in which there is no analogue of $\left\langle F_{i}^{A}\right\rangle$ and where the multiplicative term is very small. Thus our coarse-graining procedure yields an effective equation with both additive and multiplicative forcing terms $F_{i}^{A}$ and $F_{i}^{M} . F_{i}^{A}$ has a nonzero mean $\left\langle F_{i}^{A}\right\rangle$ unlike the additive, stochastic force in the $\mathrm{RFNSE}^{4}$ and $\delta F_{i}^{A}$ is much smaller than $\left\langle F_{i}^{A}\right\rangle$.

We will show below that the multiplicative forcing term generated by our coarsegraining procedure is, in some respects, similar to the effective viscosity used in the constrained Euler system [21] of She and Jackson (SJ hereafter). To make this clear we begin with a brief description of this constrained Euler system: It is a reduced system that consists of inertial-range $(\mathscr{I})$ modes, referred to as the explicit modes $v^{E}(\mathbf{k})$ by SJ. The rest of the modes, outside the reduced system, are called the implicit modes $v^{I}(\mathbf{k})$ (these lie in our ranges $\mathscr{F}$ and $\mathscr{D}$ ). In the statistically stationary state, the energy spectrum $E(k)=\frac{1}{2} \sum_{|\mathbf{k}|=k}|\mathbf{v}(\mathbf{k})|^{2}$, fluctuates in time around a well-defined mean. SJ argue that, for isotropic turbulence, the relative amplitude of the fluctuation at a given $k$ shell is $\sim 1 / \sqrt{4 \pi k^{2}}$, and thus becomes small for large $k$. So, as a first approximation, they suggest the "strong stationarity" assumption

$$
\partial_{t} E(k)=\frac{1}{2} \sum_{|\mathbf{k}|=k}[\mathbf{v}(\mathbf{k}) \cdot \mathbf{B}(-\mathbf{k})+\mathbf{v}(-\mathbf{k}) \cdot \mathbf{B}(\mathbf{k})]-2 v k^{2} E(k)=0,
$$

where $\mathbf{B}(\mathbf{k})$, a function of both explicit and implicit modes, is

$$
\begin{aligned}
& B_{j}(\mathbf{k})=B_{j}^{E}(\mathbf{k})+B_{j}^{I}(\mathbf{k}), \\
& B_{j}^{E}(\mathbf{k})=-i M_{j l m} \sum_{q} v_{l}^{E}(\mathbf{q}) v_{m}^{E}(\mathbf{k}-\mathbf{q}) .
\end{aligned}
$$

$B_{j}^{E}(\mathbf{k})$ describes interactions which involve only the explicit modes $v^{E}(\mathbf{k})$. SJ use an additional assumption about the nonlinear interactions between the implicit modes,

\footnotetext{
${ }^{3}$ While evaluating $\Delta v$ we extract $D(k)$ from the 3-point function by choosing $l, m, n$ and the values of $k_{l}, k_{m}, k_{n}$ judiciously. Possible choices are $\left(m=n \neq l, k_{n}=0\right),\left(m=n=l, k_{n}=0\right),\left(m=n \neq l, k_{m}=0\right)$, etc. ${ }^{4}$ However, it is easy to see that $\left\langle F_{i}^{A}\right\rangle$ decays rapidly with $k$ : For fixed $\mathbf{k}$ let the number of $(\mathbf{q}, \mathbf{k}-\mathbf{q})$ pairs, such that $\mathbf{q}, \mathbf{k}-\mathbf{q} \in \mathscr{F}$, be $n(k)$. It is easy to see that $n(k)$ is proportional to the volume enclosed between two spheres, each of radius $\Lambda_{L}$ and whose centres are separated by a distance $k$. Thus $n(k)=\frac{\pi}{3} \Lambda_{L}^{3}\left[2-3 x+x^{3}\right]$, with $x=\frac{k}{2 \Lambda_{L}}$. Note that $n(k)$ hits zero at $k=2 \Lambda_{L}$, which implies $\left\langle F_{i}^{A}(\mathbf{k})\right\rangle=0$, for $k>2 \Lambda_{L}$.
} 
namely, that $B_{j}^{I}(\mathbf{k})$ can be approximated as

$$
B_{j}^{I}(\mathbf{k})=\lambda(k, t) v_{j}^{E}(\mathbf{k}, t),
$$

where $\lambda(k, t)$ is real. From Eqs. (21) and(22) it follows that $\lambda(k, t)=v k^{2}-(i / 2) \psi(k, t)$, so $\psi(k, t)$ must be purely imaginary and is

$$
\psi(k)=\frac{1}{E(k)} \sum_{|\mathbf{k}|=k} \sum_{\mathbf{q}} M_{j l m}(\mathbf{k}) v_{j}^{E}(-\boldsymbol{k}) v_{l}^{E}(\mathbf{q}) v_{m}^{E}(\mathbf{k}-\mathbf{q}) .
$$

By substituting the expression for $B_{j}^{I}(\mathbf{k})$ (Eq. (23)) into Eq. (2), we get the reduced dynamical system

$$
\partial_{t} v_{j}^{E}(\mathbf{k})=-i\left[M_{j l m}(\mathbf{k}) \sum_{\mathbf{q}} v_{l}^{E}(\mathbf{q}) v_{m}^{E}(\mathbf{k}-\mathbf{q})+\psi(k) v_{j}^{E}(\mathbf{k})\right] .
$$

SJ show that a DNS of this constrained equation on a $16^{3}$ grid reproduces, quite accurately, many of the results obtained from a DNS of the $3 d \mathrm{NSE}$ on a $128^{3}$ grid. These results include the $k^{-5 / 3}$ energy spectrum, the Kolmogorov constant $C_{k}=1.88 \pm$ 0.02 , the skewness factor, and 4th- and 6th-order flatness factors, etc. Note that $\psi(k)$ is a time varying quantity and depends only on explicit modes. Also the time-averaged mean of $[-i \psi(k)]$ is found to be positive for the lowest values of $k$ and negative for larger values. $\psi(k)$ changes sign under a time-reversal transformation. Hence $[-i \psi(k)]$ can be interpreted as an effective viscosity. For small $k$, where $[-i \psi(k)]$ is positive, it acts like an effective forcing on the shells; for larger values of $k$, where it is negative, it extracts energy from the modes like the normal viscosity term in the $3 d$ NSE.

In the spirit of the SJ approximation (Eq. (23)) we write the multiplicative forcing term $T_{3}$ as (see Eqs. (4)-(12))

$$
-i M_{i m n}(\mathbf{k}) \sum_{\mathbf{q} \in \mathscr{F}, \mathbf{k}-\mathbf{q} \in \mathscr{I}}\left\langle F_{m}^{M}(\mathbf{q})\right\rangle v_{n}(\mathbf{k}-\mathbf{q}, t)=\left(\lambda_{1}(k, t)+v k^{2} / 2\right) v_{i}(\mathbf{k}, t),
$$

and

$$
-i M_{i m n}(\mathbf{k}) \sum_{\mathbf{q} \in \mathscr{D}, \mathbf{k}-\mathbf{q} \in \mathscr{I}} \delta F_{m}^{M}(\mathbf{q}, t) v_{n}(\mathbf{k}-\mathbf{q}, t)=\left(\lambda_{2}(k, t)+v k^{2} / 2\right) v_{i}(\mathbf{k}, t),
$$

where $\lambda_{1}$ and $\lambda_{2}$ are real. If we multiply Eqs. (26) and (27) by $v_{i}(-\mathbf{k})$, sum over all the $\mathbf{k}$ modes belonging to a shell with $|\mathbf{k}|=k$, and then average over a time $\tau_{U}$, we get

$$
\begin{aligned}
& \bar{\lambda}_{1}(k)+v k^{2} / 2=\frac{-i}{E(k)} \sum_{|\mathbf{k}|=k} M_{i m n}(\mathbf{k}) \sum_{\mathbf{q} \in \mathscr{F}, \mathbf{k}-\mathbf{q} \in \mathscr{I}}\left\langle F_{m}^{M}(\mathbf{q})\right\rangle\left\langle v_{i}(-\mathbf{k}) v_{n}(\mathbf{k}-\mathbf{q})\right\rangle, \\
& \bar{\lambda}_{2}(k)+v k^{2} / 2=\frac{-i}{E(k)} \sum_{|\mathbf{k}|=k} M_{i m n}(\mathbf{k}) \sum_{\mathbf{q} \in \mathscr{O}, \mathbf{k}-\mathbf{q} \in \mathscr{I}}\left\langle\delta F_{m}^{M}(\mathbf{q}) v_{i}(-\mathbf{k}) v_{n}(\mathbf{k}-\mathbf{q})\right\rangle,
\end{aligned}
$$

where $E(k)$ is energy spectrum averaged over a time $\sim \tau_{U}$. In the above equations we have made the approximation

$$
\left\langle\lambda_{1,2}(k, t) v_{i}(-\mathbf{k}, t) v_{i}(\mathbf{k}, t)\right\rangle \simeq \bar{\lambda}_{1,2}(k) E(k) .
$$


Our $\left[\bar{\lambda}_{1}(k)+v k^{2} / 2\right]$ is the analogue of the positive part of SJs $[-i \psi(k)]$ and $\left[\bar{\lambda}_{1}(k)+v k^{2} / 2\right]$ is the analogue of its negative part. Indeed it is quite plausible that $\bar{\lambda}_{1}(k)+v k^{2} / 2>0$, at least for small $k$, since the modes in $\mathscr{F}$ force those in $\mathscr{I}$ much like a negative viscosity at small $k$. A similar argument suggests that $\bar{\lambda}_{2}(k)+v k^{2} / 2<0$, at least for large $k$, because the couplings between $\mathscr{D}$ and $\mathscr{I}$ modes drain energy from the $\mathscr{I}$ modes. Thus with our coarse graining and the SJ-type approximations (Eqs. (26)-(30)) the effective equation is

$$
\begin{aligned}
\partial v_{i}(\mathbf{k}) / \partial t= & {\left[\delta v(k)+\bar{\lambda}_{1}(k)+\bar{\lambda}_{2}(k)\right] v_{i}(\mathbf{k}) } \\
& -i M_{i m n}(\mathbf{k}) \sum_{\mathbf{q}, \mathbf{k}-\mathbf{q} \in \mathscr{I}} v_{m}(\mathbf{q}) v_{n}(\mathbf{k}-\mathbf{q})+F_{i}^{A}(\mathbf{k}) .
\end{aligned}
$$

Note that, even after these approximations, there are some important differences between our effective equation (Eq. (31) and that of SJ (Eq. (25)). (1) Our effective equation has an additive forcing term $F_{i}^{A}$ which is absent in that of SJ. (2) In SJs equation $E(k)$ is held fixed (they obtain $E(k)$ by running a DNS of the $3 d \mathrm{NSE}$ ), but this is not the case in our effective equation.

Even though our coarse-graining procedure does not yield the RFNSE or the SJ effective equation, we give some representative data from our study to substantiate the points made above. Since we want to make order-of-magnitude comparisons, we give data from a DNS of the $3 d \mathrm{NSE}$ on a $16^{3}$ grid. We have verified that the additive forcing term $T_{1}(k)$ goes to zero for $k>2 \Lambda_{L}$ and the term $T_{4}(k)$ is nonzero only for $k \lesssim \Lambda_{U}$ (and zero for smaller $k$ values). For a representative $\mathbf{k}$ mode $\left(k_{x}=2, k_{y}=2, k_{z}=3\right.$ ) which lies halfway between $\Lambda_{L}$ and $\Lambda_{U}$, the term $T_{2}(k)$ (which is responsible for $\Delta v$ ) is of the same order of magnitude as the part of the $T_{3}(k)$ term which couples $\mathscr{I}$ and $\mathscr{D}$ modes. The part of the $T_{3}(k)$ term which couples $\mathscr{I}$ and $\mathscr{F}$ modes is $10^{2}$ times larger than the $T_{2}(k)$ term. For the same representative mode $\bar{\lambda}_{1}$ is $10^{3}$ times stronger than $\bar{\lambda}_{2}$, but $\bar{\lambda}_{1}(k) / \bar{\lambda}_{2}(k)$ decreases as $k$ increases, which shows that $\bar{\lambda}_{1}(k)$ is dominant when $k$ is close to $\Lambda_{L}$ whereas $\bar{\lambda}_{2}(k)$ is dominant close to $\Lambda_{U}$. Unfortunately $\bar{\lambda}_{1}$ and $\bar{\lambda}_{2}$ do not turn out to be real; the magnitudes of their real and imaginary parts are comparable over the time scales we have averaged; this we believe is a shortcoming of SJ-type approximations.

We have already noted that our coarse-graining procedure does not lead to the RFNSE. However, a very similar coarse-graining procedure has been used to map the Kuramoto-Sivashinski equation onto the KPZ equation both in one $[18,19]$ and two dimensionsi [20]. It is instructive to try to understand why the $\mathrm{KS} \rightarrow \mathrm{KPZ}$ coarse-graining works whereas the $3 d \mathrm{NSE} \rightarrow$ RFNSE does not. The principal difference is that, for the $3 d \mathrm{NSE}$ we must sum over the modes in $\mathscr{F}$; as a result the additive forcing term $F_{i}^{A}$ develops a nonzero mean. Also the multiplicative forcing is not negligible here as in the $\mathrm{KS} \rightarrow \mathrm{KPZ}$ case. In the KS equation there is no external forcing. The driving comes from the linearly unstable modes of the system (Fig. 5). The strongest forcing comes from around the peak at $k_{\max }$. As $k$ increases the modes fluctuate faster. Since one is interested in the behaviour of small $k$ modes, all the modes upto say $k_{1}$ are 


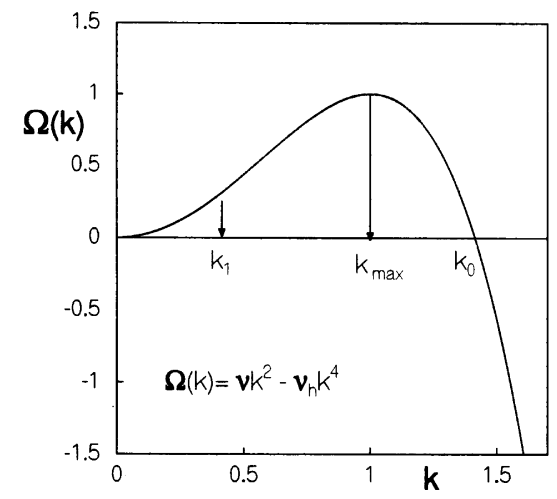

Fig. 5. The growth rate $\Omega(k)$ for a mode with wave vector $k$ in the linearised KS equation.

integrated out. The forcing because of the fast, high- $k$ modes shows up as an additive noise on the small- $k$ modes. Such an additive noise also results from our coarse graining of the $3 d \mathrm{NSE}$ but it is much smaller than the additive and multiplicative terms that arise because of a summation of the modes in $\mathscr{F}$ as described above.

\section{Acknowledgements}

We thank C. Das, C. Jayaprakash, A. Pande, and S. Ramaswamy for discussions, and SERC (IISc, Bangalore) for computational resources. One of us (AS) thanks CSIR (India) and JNCASR (India) for supporting work in India and the NSERC (Canada) for supporting work in Canada.

\section{References}

[1] A. Sain, Manu, R. Pandit, Phys. Rev. Lett. 81 (1998) 4377.

[2] A.N. Kolmogorov, C.R. Acad. Sci. USSR 30 (1941) 301.

[3] U. Frisch, Turbulence: The Legacy of A.N. Kolmogorov, Cambridge Univ. Press, Cambridge, 1995.

[4] K.R. Sreenivasan, R. Antonia, Annu. Rev. Fluid Mech. 29 (1997) 435.

[5] S.K. Dhar, A. Sain, A. Pande, R. Pandit, Pramana: J. Phys. 48 (1997) 325 (Special issue on Nonlinearity and Chaos in Physical Sciences).

[6] N. Cao, S. Chen, K.R. Sreenivasan, Phys. Rev. Lett. 77 (1996) 3799.

[7] Z.S. She, E. Leveque, Phys. Rev. Lett. 72 (1994) 336.

[8] C. DeDominicis, P.C. Martin, Phys. Rev. A 19 (1979) 419.

[9] V. Yakhot, S.A. Orszag, Phys. Rev. Lett. 57 (1986) 1722.

[10] C.-Y. Mou, P.B. Weichman, Phys. Rev. Lett. 70 (1993) 1101.

[11] G.L. Eyink, Phys. Fluids 6 (1994) 3063.

[12] V. Yakhot, S.A. Orszag, R. Panda, J. Sci. Comput. 3 (1988) 139.

[13] E.D. Siggia, J. Fluid Mech 107 (1981) 375.

[14] Z.S. She, E. Jackson, S.A. Orszag, Nature 344 (1990) 226.

[15] S. Douady, Y. Couder, M.E. Brachet, Phys. Rev. Lett. 67 (1991) 983.

[16] R. Benzi et al., Phys. Rev. E 48 (1993) R29.

[17] S.K. Dhar, A. Sain, R. Pandit, Phys. Rev. Lett. 78 (1997) 2964. 
[18] S. Zaleski, Physica D 34 (1989) 427.

[19] F. Hayot, C. Jayaprakash, Ch. Josserand, Phys. Rev. E 47 (1993) 911.

[20] C. Jayaprakash, F. Hayot, R. Pandit, Phys. Rev. Lett. 71 (1993) 15.

[21] Z.S. She, E. Jackson, Phys. Rev. Lett. 70 (1993) 1255.

[22] A. Sain, Ph.D. thesis, Indian Institute of Science, Bangalore, 1999.

[23] N. Cao, S. Chen, Z.S. She, Phys. Rev. Lett. 77 (1996) 3711.

[24] D. Forster, D.R. Nelson, M.J. Stephen, Phys. Rev. A 16 (1977) 732.

[25] J.K. Bhattacharjee, J. Phys. A 21 (1988) L551.

[26] C.-Y. Mou, P.B. Weichman, Phys. Rev. E 52 (1995) 3738.

[27] B. Dernoncourt, J.F. Pinton, S. Fauve, Physica D 117 (1998) 181. 